\title{
The endemic radiodonts of the Cambrian Stage 4 Guanshan Biota of South China
}

De-Guang Jiao, Stephen Pates, Rudy Lerosey-Aubril, Javier Ortega-Hernández, Jie Yang, Tian Lan, and Xi-Guang Zhang

Acta Palaeontologica Polonica 66 (2), 2021: 255-274 doi:https://doi.org/10.4202/app.00870.2020

The Guanshan Biota (South China, Cambrian, Stage 4) contains a diverse assemblage of biomineralizing and non-biomineralizing animals. Sitting temporally between the Stage 3 Chengjiang and Wuliuan Kaili Biotas, the Guanshan Biota contains numerous fossil organisms that are exclusive to this exceptional deposit. The Guanshan Konservat-Lagerstätte is also unusual amongst Cambrian strata that preserve non-biomineralized material, as it was deposited in a relatively shallow water setting. In this contribution we double the diversity of radiodonts known from the Guanshan Biota from two to four, and describe the second species of Paranomalocaris. In addition, we report the first tamisiocaridid from South China, and confirm the presence of a tetraradial oral cone bearing small and large plates in "Anomalocaris" kunmingensis, the most abundant radiodont from the deposit. All four radiodont species, and three genera, are apparently endemic to the Guanshan Biota. When considered in the wider context of geographically and temporally comparable radiodont faunas, endemism in Guanshan radiodonts is most likely a consequence of the shallower and more proximal environment in which they lived. The strong coupling of free-swimming radiodonts and benthic communities underlines the complex relationship between the palaeobiogeographic and environmental distributions of prey and predators. This local adaptation of radiodonts to their prey is highlighted by the frontal appendage morphology of the two species of Paranomalocaris, apparently specialised to different feeding modes, while the recognition of the limited geographic range of some radiodont faunas highlights the importance of exploring as many deposits as possible to fully understand this group.

Key words: Amplectobeluidae, Anomalocarididae, Tamisiocarididae, Paranomalocaris , Burgess Shale-type exceptional preservation, shallow water, Cambrian, China.

De-Guang Jiao [103256437@qq.com], Yuxi Normal University, 134 Phoenix Road, Yuxi, Yunnan, 653100, China: Key Laboratory for Palaeobiology, Yunnan University, Kunming, 650091, China; Yunnan Land and Resources Vocational College, Kunming, 650091, China. Stephen Pates [sp587@cam.ac.uk], 
Museum of Comparative Zoology and Department of Organismic and

Evolutionary Biology, Harvard University, Cambridge, MA 02138, USA;

Department of Zoology, University of Cambridge, Downing Street,

Cambridge, CB2 3EJ, UK. Rudy Lerosey-Aubril [rudy_lerosey@fas.harvard.edu

], Museum of Comparative Zoology and Department of Organismic

and Evolutionary Biology, Harvard University, Cambridge, MA

02138, USA. Javier Ortega-Hernández [jortegahernandez@ fas.harvard.edu]

(corresponding author), Museum of Comparative Zoology and Department of

Organismic and Evolutionary Biology, Harvard University, Cambridge, MA 02138,

USA. Jie Yang [yangjie@ynu.edu.cn] and Xi-Guang Zhang [xzhang@ynu.edu]

(corresponding author), Key Laboratory for Palaeobiology and MEC International

Joint Laboratory for Palaeoenvironment, Yunnan University, Kunming, 650091,

China; Yunnan Land and Resources Vocational College, Kunming, 650091, China.

Tian Lan [lantianing@sina.com] College of Resources and Environmental Engineering, Guizhou University, Guiyang 550003, China.

This is an open-access article distributed under the terms of the Creative Commons Attribution License (for details please see creativecommons.org), which permits unrestricted use, distribution, and reproduction in any medium, provided the original author and source are credited.

FDF 